\title{
Application of a grid numerical method to calculate state-selective cross sections for electron capture in $\mathrm{Be}^{4+}+\mathrm{H}(1 s)$ collisions
}

\author{
A. Jorge, J. Suárez, Clara Illescas, L. F. Errea, and L. Méndez \\ Laboratorio Asociado al CIEMAT de Física Atómica y Molecular en Plasmas de Fusión \\ and Departamento de Química, módulo 13, Universidad Autónoma de Madrid, Cantoblanco E-28049 Madrid, Spain
}

(Received 14 July 2016; published 19 September 2016)

\begin{abstract}
Charge-transfer $n$ partial cross sections have been calculated for collisions of $\mathrm{Be}^{4+}$ with $\mathrm{H}(1 s)$ by means of a versatile lattice method that is applicable in a wide energy range (between 1 and $500 \mathrm{keV} / \mathrm{u}$ ). The cross sections, which include up to the high-lying $n=8$ level, are compared to existing semiclassical calculations in order to quantify the accuracy of the results. The reliability of the lattice method at high impact energies is confirmed by comparison with classical trajectory Monte Carlo calculations. It is found that the $n$ partial cross sections larger than $10^{-18} \mathrm{~cm}^{2}$, calculated using the lattice method, agree with differences smaller than $15 \%$ with those from the method considered the most accurate at each energy. The calculation yields as well accurate total electron capture cross sections, which are studied in detail at $E=100 \mathrm{keV} / \mathrm{u}$ to obtain a converged value.
\end{abstract}

DOI: 10.1103/PhysRevA.94.032707

\section{INTRODUCTION}

Collisions involving $\mathrm{Be}^{q+}$ ions are especially relevant because Be will be used as the armor material of the first wall of the International Thermonuclear Experimental Reactor (ITER) (see [1]) and an ITER-like wall is already under operation in the Joint European Torus (JET) [2,3]. Chemical and physical erosion of the first wall releases beryllium atoms and several molecular species, which eventually lead to the presence of fully stripped beryllium ions in the plasma core. The diagnostics of impurity density and temperature in the plasma core is carried out by applying the charge exchange recombination spectroscopy (CXRS) technique [4], where a fast beam of $\mathrm{H}$ atoms (100 keV in ITER [5]) collides with the impurity ions $X^{q+}$, leading to the electron capture (EC) reactions:

$$
X^{q+}+\mathrm{H} \rightarrow X^{(q-1)+*}+\mathrm{H}^{+} .
$$

The diagnostics is based on the emission, usually in the visible spectrum (see [6]), of the excited ions, $X^{(q-1)+*}$.

The application of the CXRS diagnostics requires the knowledge of state-resolved EC cross sections (1), which are in general difficult to measure, and, in particular, only theoretical data are available for collisions involving $\mathrm{Be}^{q+}$ ions. The relevance of EC in fusion research has motivated many works [7-21] applying very different methods, but despite the apparent simplicity of monoelectronic systems, significant discrepancies remain between the results. While close-coupling and distorted-wave methods are very accurate in the low $(E<25 \mathrm{keV} / \mathrm{u})$ and high $(E>1000 \mathrm{keV} / \mathrm{u})$ energy range, respectively, the cross sections for the high- $n$ capture levels are far from been converged in the intermediate energy domain that characterizes the probe beam.

On the other hand, the precision of the calculated cross sections for heavy-particle collisions is in general unknown, and the quantification of the uncertainties associated with these cross sections is a difficult task that has not been undertaken hitherto. The uncertainties associated with cross section calculations are required by data users and the efforts to quantify the uncertainties of the atomic data have been lead by the Atomic and Molecular Data Unit, Nuclear Data Section, of the International Atomic Energy Agency (see [22] and the recent review on uncertainty estimates for both structure and collision calculations of Ghung et al. [23]). However, although the necessity of including the uncertainty estimates of the collisional data is generally accepted (see the editorial policy of Physical Review A [24]), only a few works on electronic collisions (see, e.g., [25]) have provided the uncertainties of the calculated data.

In this work we have calculated $n$ partial cross sections for the processes

$$
\mathrm{Be}^{4+}+\mathrm{H}(1 s) \rightarrow \mathrm{Be}^{3+}(n)+\mathrm{H}^{+}
$$

in the broad energy range $1 \mathrm{keV} / \mathrm{u} \leqslant E \leqslant 500 \mathrm{keV} / \mathrm{u}$, and up to $n=8$, which is a range of energies and exit channels that cannot be accurately covered by either close-coupling or perturbative methods. These calculations have been performed using a single semiclassical method, in which we solve numerically the time-dependent Schrödinger equation with the GridTDSE package (GTDSE) [26]. The GTDSE package was originally designed for nuclear processes in molecular dynamics, and has been modified to treat electron dynamics. Specifically, it has been recently applied to treat ionization processes [27]. The EC reaction (2) has been studied in the past by Minami et al. [19] with a lattice method, providing partial cross sections for $n \leqslant 5$. However, it must be noted that the CXRS diagnostics for Be (IV) works in the visible emission, which corresponds to the transitions $n=6 \rightarrow n=5$ and $n=8 \rightarrow n=6$ of $\mathrm{Be}^{3+}$. Accurate values of partial cross sections into levels with $n>5$, as proposed in the present paper, are therefore key input values for the realistic performance of CXRS experiments. One must note that the calculation of EC cross sections into excited levels requires the use of large lattices that involve exceptional computational resources.

The second aim of the present calculation is to gauge the applicability of the GTDSE method at relatively low collision energies $(E=1 \mathrm{keV} / \mathrm{u})$, not considered in [19], by comparison with state-of-the-art molecular-orbital-closecoupling (MOCC) [16] and recent atomic-orbital-closecoupling (AOCC) calculations [20]. This comparison yields 
valuable information on the systematic application of the the lattice methods to treat low-energy collisions. At intermediate energies $(E \approx 30 \mathrm{keV} / \mathrm{u})$, the lattice GTDSE cross sections are compared with AOCC data in order to estimate the uncertainties of these calculations. Close-coupling methods are based on the expansion on complete sets, provided that they include both bound and continuum states. In practice, the electronic continuum is represented by a set of $L^{2}$ integrable functions, called pseudostates, but the choice of these pseudostates introduces an inherent uncertainty. On the other hand, in the lattice calculations the collisional wave function is evaluated at the nodes of a 3-dimensional grid, and the precision is determined by the extension and density of the grid. Since the uncertainties are very different in nature, the comparison between probabilities and cross sections obtained with close-coupling and lattice methods provides an estimate of their intrinsic uncertainties, which is difficult to obtain from the application of a single methodology.

At higher energies $(E \approx 100 \mathrm{keV} / \mathrm{u})$, the close-coupling methods become unpractical given the large number of states (bound and continuum) required. At these energies, the stateselected EC cross sections are often obtained by applying the classical trajectory Monte Carlo (CTMC) method with a microcanonical distribution [28] for describing the initial state of the hydrogen atom. The suitability of the CTMC method to represent EC into highly excited levels is hindered by the wellknown inadequacy of the initial microcanonical distribution to accurately reproduce the quantal electron distribution. In order to overcome this limitation, improved initial classical distributions, with a better agreement of the radial distribution with the quantal one, have been proposed in the past [29-31], which yield practically the same results. More recently, an alternative distribution has been proposed by Cariatore et al. [32]. It has been shown [33] that the distribution of Ref. [32] is not stable and cannot be applied for excited states. The results of applying this distribution for collisions of multicharged ions with $\mathrm{H}(1 s)$ have been compared with previous results in both the Comment [33] and the Reply [34], where it was found that at energies $E \approx 100 \mathrm{keV} / \mathrm{u}$ the distribution of Ref. [32] leads to EC partial cross sections practically identical to those calculated with the microcanonical distribution. As mentioned in [19], the comparison between the results of the CTMC, with alternative initial distributions, and the numerical results is valuable in order to discuss the accuracy of the cross sections. In this respect, we include in the present work CTMC calculations with both the microcanonical and the hydrogenic [29] distributions.

Concerning the total electron capture cross sections of $\mathrm{Be}^{4+}+\mathrm{H}$ collisions, there is a remarkable disagreement in the existing databases at $E=100 \mathrm{keV} / \mathrm{u}$, where the splitting between the available results is around $30 \%$ of the total cross section. This disagreement is particularly relevant in fusion plasmas since it appears at the energy of the ITER diagnostics beam. In this work we discuss the limitations of previous works and we recommend a value of the total cross section at this energy.

The paper is organized as follows: In Sec. II we explain the lattice method employed. In Sec. III we discuss the partial cross sections at four representative impact energies: 1, 30, 100 , and $500 \mathrm{keV} / \mathrm{u}$. The total EC cross section is considered in Sec. IV and a brief summary is presented in Sec. V. Atomic units are employed unless otherwise stated.

\section{THE GTDSE METHOD}

At the energies considered in this work one can employ the semiclassical impact parameter approximation, where the nuclei follow rectilinear trajectories defined by $\boldsymbol{R}(t)=\boldsymbol{b}+$ $\boldsymbol{v} t$, with $\boldsymbol{b}$ the impact parameter and $\boldsymbol{v}$ the relative velocity. The electronic motion is then described by the wave function $\Psi(\boldsymbol{r}, t)$, solution of the semiclassical equation:

$$
\left[H_{\mathrm{el}}-\left.i \frac{\partial}{\partial t}\right|_{\boldsymbol{r}}\right] \Psi=0
$$

with the electronic Hamiltonian:

$$
H_{\mathrm{el}}(\boldsymbol{r}, \boldsymbol{R}(t))=-\frac{1}{2} \nabla_{\boldsymbol{r}}^{2}+V_{\mathrm{H}}+V_{\mathrm{Be}},
$$

where $\boldsymbol{r}$ is the electron position vector.

We have adapted the parallel GridTDSE package [26] to solve numerically Eq. (3) for not-bound systems and a time-dependent potential operator. In the present application, the wave function $\Psi$ is evaluated at the points of a $3 \mathrm{D}$ Cartesian lattice, with the origin on the Be nucleus in order to evaluate asymptotically the capture processes. The lattice representation of the electronic wave function is a vector $\boldsymbol{\Psi}$, solution of the matrix equation

$$
\boldsymbol{H} \boldsymbol{\Psi}=(\boldsymbol{T}+\boldsymbol{V}) \boldsymbol{\Psi}=i \dot{\boldsymbol{\Psi}} .
$$

$\boldsymbol{V}$ is a diagonal matrix that stores the values of the potential $V_{\mathrm{Be}}+V_{\mathrm{H}}$ at the grid points, while the kinetic energy matrix, $\boldsymbol{T}$, is a nondiagonal sparse matrix that is obtained by applying the finite-difference method (see [35]) considering a stencil of $n_{s}$ neighboring grid points. Equation (5) is time integrated by iteratively applying the second-order difference method (SOD) with small time steps (i.e., of the order of $10^{-2}$ a.u.), during which the electron-target Coulomb attraction is approximately constant. The initial condition at $t_{0}$ is the grid representation of the traveling $\mathrm{H}(1 s)$ orbital,

$$
\Psi\left(\boldsymbol{r}, t_{0}\right)=\varphi_{1 s}^{\mathrm{H}}\left[\boldsymbol{r}-\boldsymbol{R}\left(t_{0}\right)\right] \exp \left(-i \boldsymbol{v} \cdot \boldsymbol{r}-i / 2 v^{2} t_{0}\right) .
$$

The extension of the grid is taken as $-L_{\max } \leqslant q \leqslant L_{\max }, q=$ $x, z$, and $0 \leqslant y \leqslant L_{\max }$, where we have taken advantage of the symmetry of the Hamiltonian upon reflection in the collision plane $(X Z)$. The parallelization strategy followed in GridTDSE is particularly efficient for Cartesian coordinates due to the fact that the Hamiltonian matrix is highly sparse, allowing the consideration of the massive grids $\left(\approx 10^{8}\right.$ grid points $)$ characteristic of collisional processes. The present calculations were carried out with a broad box of $L_{\max }=40 a_{0}$. Although it involves vast memory allocations (on the order of $256 \mathrm{~Gb}$ ) that can only be assumed with the optimized parallelization strategy, such an extension allows the description of the $\mathrm{Be}^{3+}$ orbitals up to the quantum level $n \leqslant 8$. On the other hand, the convergence of the second derivatives is rapidly reached in Cartesian coordinates with a stencil of $n_{s}=15$ points. The precision of the provided cross sections has been tested with several grid densities: G1, with $\Delta_{q}=q_{i+1}-q_{i}=0.2 a_{0}$; G2, with $\Delta_{q}=0.137 a_{0}$. For the specific case of capture into $n=1,2$ levels, where the convergence is particularly slow, we have additionally performed calculations with grids G3 $\left(\Delta_{q}=0.1 a_{0}\right)$ 
TABLE I. Soft-core parameters $\epsilon_{\mathrm{H}}, \epsilon_{\mathrm{Be}}$ [Eq. (7)] employed for different grid densities. Numbers in square brackets indicate power of 10 .

\begin{tabular}{lcc}
\hline \hline$\Delta_{q}$ (a.u.) & $\epsilon_{\mathrm{H}}$ & $\epsilon_{\mathrm{Be}}$ \\
\hline $0.2(\mathrm{G} 1)$ & $3.65[-3]$ & $5.40[-3]$ \\
$0.137(\mathrm{G} 2)$ & $1.70[-3]$ & $2.22[-3]$ \\
$0.1(\mathrm{G} 3)$ & $7.98[-4]$ & $1.08[-3]$ \\
$0.05(\mathrm{G} 4)$ & $1.75[-4]$ & $2.29[-4]$ \\
\hline \hline
\end{tabular}

and G4 $\left(\Delta_{q}=0.05 a_{0}\right)$. Nevertheless, for the sake of the memory allocation, the extension of the grid in these cases has been substantially reduced, which allows the calculation of the capture probabilities into $n \leqslant 3$. As a final remark, according to the uncertainty principle, the time step employed in the time integration is directly related to the grid spacing as $\Delta t<1 / E_{\max }$, with $E_{\max }=\pi^{2} /\left(2 \Delta_{q}^{2}\right)$ [36]. Therefore, as the description of the wave function in the spatial coordinates improves with increasing density, shorter time steps must be employed.

In practice, as in previous numerical treatments [19], a soft-core approximation is introduced to allow the integration near the Coulomb singularity. The potentials are

$$
\begin{aligned}
V_{\mathrm{H}}(\boldsymbol{r}, \boldsymbol{R}(t)) & =-\frac{1}{\left(|\boldsymbol{r}-\boldsymbol{R}|^{2}+\epsilon_{\mathrm{H}}\right)^{1 / 2}}, \\
V_{\mathrm{Be}}(\boldsymbol{r}) & =-\frac{4}{\left(r^{2}+\epsilon_{\mathrm{Be}}\right)^{1 / 2}},
\end{aligned}
$$

and the soft-core parameters $\epsilon_{\mathrm{H}, \mathrm{Be}} \ll \Delta_{q}$ have been optimized by fitting the atomic energies for each grid density (Table I), with deviations smaller than $1 \%$ from the exact energies. In order to avoid unphysical reflections at the walls of the box, we have introduced the mask function [27,37]:

$$
M(\boldsymbol{r})=\prod_{i=1,3} \begin{cases}\exp \left\{-\alpha\left(\left|q_{i}\right|-L_{\max }+\delta\right)^{2}\right\}, & \text { if } L_{\max }-\left|q_{i}\right|<\delta \\ 1, & \text { elsewhere, }\end{cases}
$$

with $\delta=3.0 a_{0}$ and $\alpha=0.002 a_{0}^{-2}$. As pointed in previous works [38,39], mask functions used in grid schemes are directly related to complex absorbing potentials. With the origin of coordinates placed at the projectile, the mask function completely absorbs the part of the wave function that correlates asymptotically to ionization and target bound states, while only those capture states with the electronic density confined inside the box can be properly described.

\section{ELECTRON CAPTURE PARTIAL CROSS SECTIONS}

\section{A. $E=1 \mathrm{keV} / \mathrm{u}$}

At $E=1 \mathrm{keV} / \mathrm{u}$, only the levels $\operatorname{Be}^{3+}(n=3,4)$ are significantly populated in the reaction (2), while the partial cross sections for populating the levels $n=2$ and $n=5$ are 100 times smaller than those of the dominant channels (see Ref. [16]). The MOCC calculations (see Table II) converge to cross section values that agree with the large-scale AOCC calculation of Ref. [20]. In this table, the MOCC (18) calculations employ a minimal 18-term basis set that includes the molecular channels dissociating into $\mathrm{Be}^{3+}(n=3,4)$, and $\operatorname{MOCC}(88,96)$ are the two extended bases of Refs. $[15,16]$. Since both MOCC

TABLE II. Total cross sections in $10^{-16} \mathrm{~cm}^{2}$ for reactions (2) at $E=1 \mathrm{keV} / \mathrm{u}$. The number of basis functions of the close-coupling calculations is indicated in brackets.

\begin{tabular}{lcc}
\hline \hline Calculation & $n=3$ & $n=4$ \\
\hline MOCC (17) [15] & 34.7 & 2.45 \\
MOCC (88) [16] & 34.5 & 3.17 \\
MOCC (96) [15] & 34.4 & 3.11 \\
AOCC (170) [20] & 34.4 & 3.10 \\
GTDSE & 33.2 & 3.27 \\
\hline \hline
\end{tabular}

and AOCC computational procedures are very different, the agreement indicates that the absolute uncertainties of these cross sections are smaller than $\pm 10^{-17} \mathrm{~cm}^{2}$. The validity of the eikonal approximation for the collision (2) has been discussed in Ref. [15] by comparing eikonal and full quantum mechanical calculations. This comparison at $E=250 \mathrm{eV} / \mathrm{u}$ shows that the differences for the total EC cross section and for the $n=3$ partial cross section are on the order of $1 \%$, and on the order of $5 \%$ for the partial cross section for populating the level $\mathrm{Be}^{3+}(n=4)$. Since the eikonal approximation becomes more accurate as $E$ increases, the uncertainty associated with the use to the eikonal approximation is expected to be smaller than $1 \%$ at $E \gtrsim 1 \mathrm{keV} / \mathrm{u}$ for the dominant channels.

The GTDSE calculations for grids G1 and G3 yield indistinguishable partial cross sections that disagree from the close-coupling ones in about 3\%-6\%. To further analyze the workings of the GTDSE calculation, we compare in Fig. 1 the EC opacity functions $b P_{n}^{\mathrm{EC}}(b)$, obtained using MOCC and GTDSE, where

$$
P_{n}^{\mathrm{EC}}=\lim _{t \rightarrow+\infty} \sum_{l, m}\left|\left\langle\phi_{n l m}^{\mathrm{Be}} \mid \Psi\right\rangle\right|^{2} .
$$

One can note that the GTDSE calculation correctly reproduces the oscillations of the transition probability with small differences in the height of the maxima. This agreement indicates that both results are consistent with the same collision mechanism. Moreover, we have checked that the eigenvalues of the molecular Hamiltonian obtained in the grid representation agree with the energies of the molecular orbitals. In particular, the gap between the electronic energies of the orbitals $4 f \sigma$ and $3 d \sigma$ in the pseudocrossing between them is 0.0985 hartrees (GTDSE) and 0.0984 hartrees (MOCC), which is particularly relevant because the transitions in this pseudocrossing furnish the main mechanism of the EC process at low velocities. 


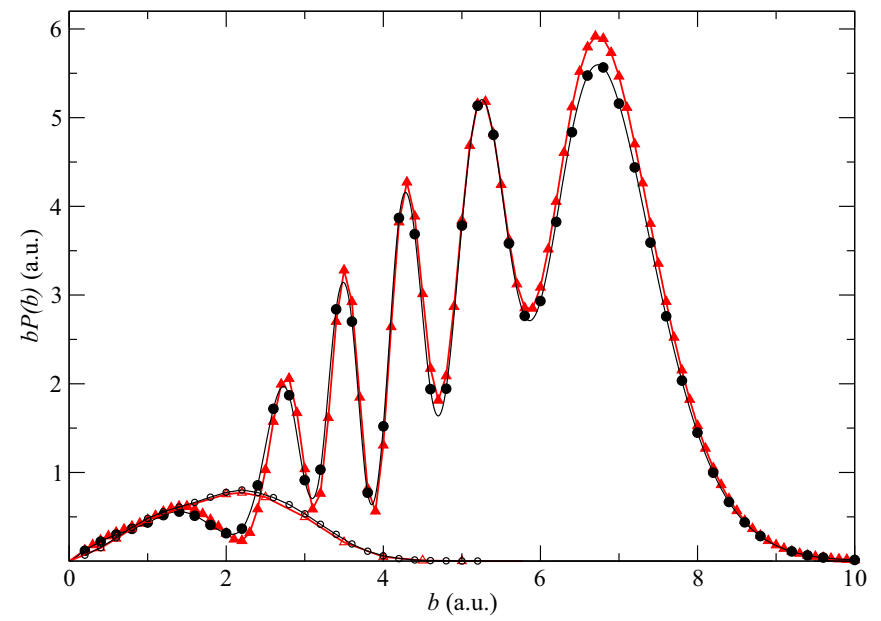

FIG. 1. Transition probability times the impact parameter [Eq. (8)] for electron capture in $\mathrm{Be}^{4+}+\mathrm{H}(1 s)$ collisions at $E=1 \mathrm{keV} / \mathrm{u} . b P_{3}^{\mathrm{EC}}:(\bullet-\bullet), \operatorname{GTDSE} ;(-\boldsymbol{\Delta}-), 88$-state MOCC. $b P_{4}^{\mathrm{EC}}:(-\circ-)$, GTDSE; $(-\triangle-), 88$-state MOCC.

\section{B. $E=30 \mathrm{keV} / \mathrm{u}$}

At $E=30 \mathrm{keV} / \mathrm{u}$ the ionization cross section starts to be relevant, and the MOCC method, without pseudostates, is not appropriate as the ionization flux populates the most energetic molecular channels, which are correlated to high- $n$ capture levels [40]. In fact, calculations at this energy were not reported in Ref. [16]. Igenbergs et al. [20,41] have carried out AOCC calculations with different basis sets that indicate that the EC total cross section has converged within $\pm 0.4 \times 10^{-16} \mathrm{~cm}^{2}$, and the partial cross sections reported in [41] for the largest basis set (170 orbitals that include 42 united atom orbitals to describe the continuum). Partial cross sections from $n=$ 1 to $n=8$ are presented in Table III. The present GTDSE results show an excellent agreement with those of Igenbergs et al. $[20,41]$, as can be noted in Table III and Fig. 2, with the exception of the partial cross section for $\mathrm{EC}$ into $\mathrm{Be}^{3+}(n=8)$, which is probably overpopulated in the AOCC calculation.

\section{C. $E=100 \mathrm{keV} / \mathrm{u}$}

We display in Fig. 3 the $n$ partial cross sections at $E=$ $100 \mathrm{keV} / \mathrm{u}$, where the unphysical behavior of the AOCC cross sections for $n>5$ is clear, probably due to an incorrect trapping of the flux leading to ionization and electron excitation in

TABLE III. Total cross sections in $10^{-16} \mathrm{~cm}^{2}$ for reactions (2) at $E=30 \mathrm{keV} / \mathrm{u}$.

\begin{tabular}{lcc}
\hline \hline$n$ & AOCC (170) [20] & GTDSE \\
\hline 1 & $3.0 \times 10^{-5}$ & $3.6 \times 10^{-5}$ \\
2 & 1.17 & 1.19 \\
3 & 11.1 & 11.1 \\
4 & 6.29 & 6.67 \\
5 & 2.51 & 2.76 \\
6 & 1.14 & 1.25 \\
7 & 0.64 & 0.64 \\
8 & 0.58 & 0.40 \\
\hline \hline
\end{tabular}

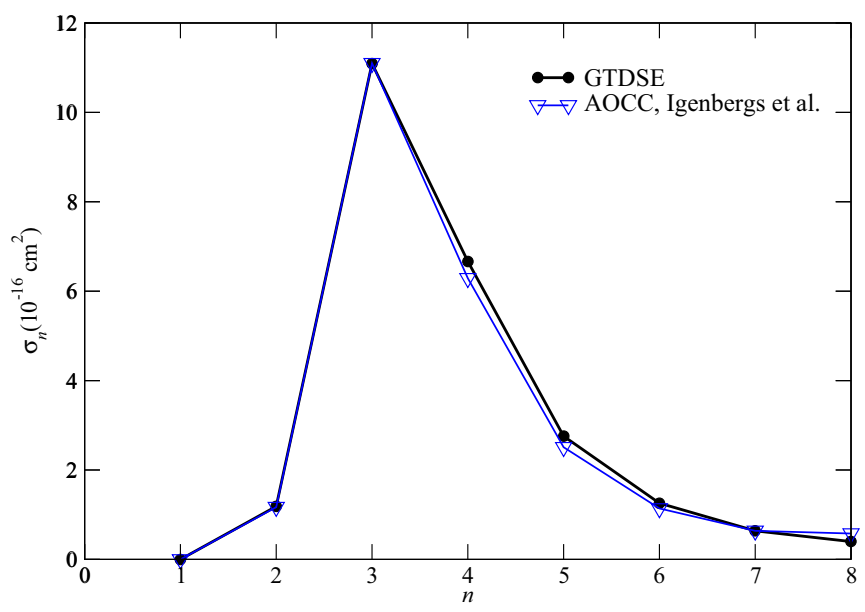

FIG. 2. $n$-partial cross sections for $\mathrm{EC}$ in $\mathrm{Be}^{4+}+\mathrm{H}(1 s)$ collisions at $E=30 \mathrm{keV} /$ u. (•), GTDSE calculation, $\nabla$, AOCC calculations of Refs. [20,41].

the most excited levels of the basis set. We include in this figure CTMC results obtained using both the microcanonical and the hydrogenic distributions, as explained in [17]. We find a general good agreement between GTDSE and CTMC-hydrogenic cross sections, while the microcanonical distribution leads to an important overestimation of the cross sections for $n \geqslant 3$. We present a more detailed comparison of GTDSE and CTMChydrogenic partial cross sections in Table IV. To ensure the convergence of the CTMC calculation we have calculated the cross sections with $10^{5}$ and $5 \times 10^{5}$ electron trajectories and we have found differences smaller than $5 \times 10^{-19} \mathrm{~cm}^{2}$. The convergence of the GTDSE calculation is shown in Table IV varying the density of the grid $(\mathrm{G} 1, \mathrm{G} 2$, and G3). It is clear that the calculations provide converged values with the exception of the very small cross section for capture into the ground state, although the value obtained with the grid G3 is close to that from the AOCC method [41] $\left(1.0 \times 10^{-19} \mathrm{~cm}^{2}\right)$. For $n \geqslant 3$ the GTDSE and the CTMC calculations agree with differences smaller than $13 \%$ of the cross sections. As has been explained in previous works $[42,43]$, the CTMC-hydrogenic

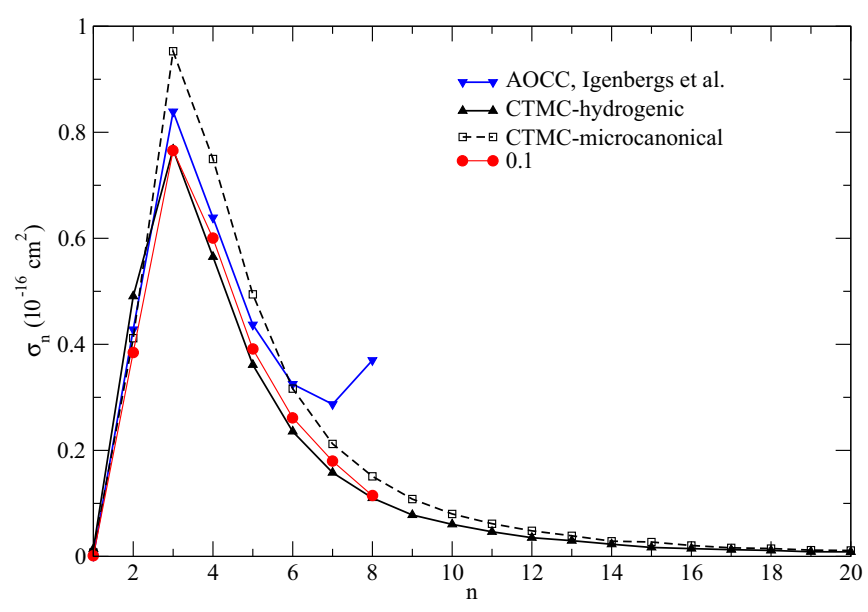

FIG. 3. n-partial cross sections for $\mathrm{EC}$ in $\mathrm{Be}^{4+}+\mathrm{H}(1 s)$ collisions at $E=100 \mathrm{keV} / \mathrm{u}$. 
TABLE IV. Total cross sections in $10^{-16} \mathrm{~cm}^{2}$ for reactions (2) at $E=100 \mathrm{keV} / \mathrm{u}$.

\begin{tabular}{lllll}
\hline \hline$n$ & CTMC & GTDSE (G1) & GTDSE (G2) & GTDSE (G3) \\
\hline 1 & 0.014 & $3.6 \times 10^{-4}$ & $3.3 \times 10^{-4}$ & $9.1 \times 10^{-4}$ \\
2 & 0.49 & 0.39 & 0.39 & 0.38 \\
3 & 0.77 & 0.77 & 0.77 & 0.77 \\
4 & 0.57 & 0.58 & 0.60 & 0.60 \\
5 & 0.36 & 0.40 & 0.39 & 0.39 \\
6 & 0.23 & 0.26 & 0.26 & 0.26 \\
7 & 0.16 & 0.18 & 0.17 & \\
8 & 0.11 & 0.12 & 0.11 & \\
\hline \hline
\end{tabular}

is not appropriate to reproduce processes into low- $n$ capture levels, and in the particular case of $\mathrm{Be}^{4+}+\mathrm{H}(1 s)$ it yields overestimated $n=1,2$ capture cross sections with regard to both GTDSE and AOCC values.

\section{D. $E=500 \mathrm{keV} / \mathrm{u}$}

In order to check the applicability of the GTDSE method at very high energies we have calculated the $n$ partial EC cross sections at $E=500 \mathrm{keV} / \mathrm{u}$. Our results are plotted in Fig. 4, together with the results from CTMC, AOCC [20], and the eikonal impulse approximation (EIA) [44,45]. The partial cross sections of Minami et al. [19], taken from their Fig. 7, are practically identical to our G3 results of Fig. 4. One can note in this plot that the CTMC-microcanonical calculation overestimates the cross sections compared to those from the other methods; this overestimation is larger for the CTMC-hydrogenic, not shown in this figure for clarity. The GTDSE calculation converges slowly because very dense grids are required in order to reproduce the fast oscillation of the plane wave associated to the electron translation motion. Accordingly, large memory is needed to store the wave function and the Hamiltonian matrix along the trajectory. Specifically, with a memory of $256 \mathrm{~Gb}$, we can employ the grid G4 with an extension (in atomic units) $-11 \leqslant x \leqslant 11$,

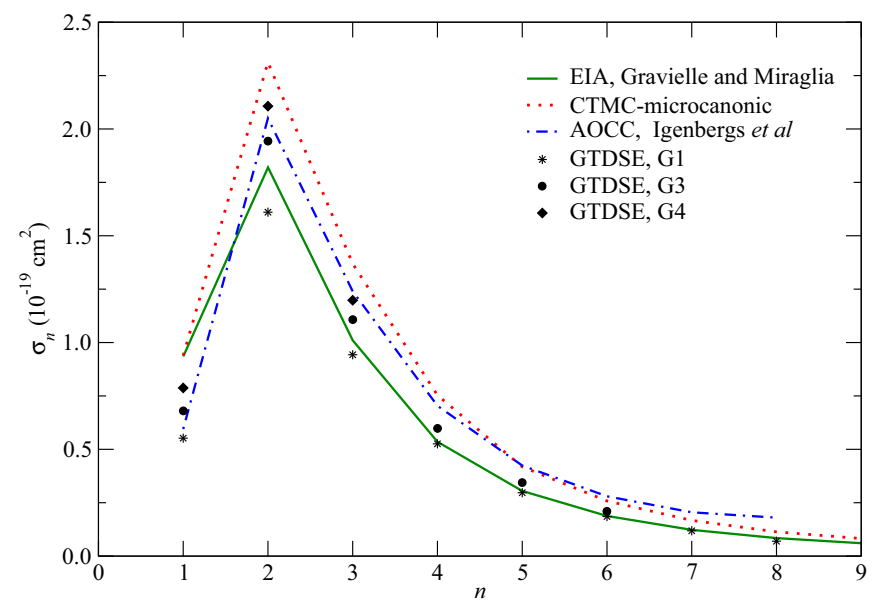

FIG. 4. $n$-partial cross sections for $\mathrm{EC}$ in $\mathrm{Be}^{4+}+\mathrm{H}(1 s)$ collisions at $E=500 \mathrm{keV} / \mathrm{u}$. Present GTDSE and CTMC calculations are compared with previous EIA [44,45] and AOCC [41] results, as indicated in the figure.
TABLE V. Total cross section for populating $\operatorname{Be}^{3+}(n=1)$ in reaction (2) at $E=500 \mathrm{keV} / \mathrm{u}$, as a function of the grid spacing $\Delta_{q}$, compared with the result of the EIA method [44].

\begin{tabular}{lc}
\hline \hline$\Delta_{q}\left(\mathrm{a}_{0}\right)$ & Cross section $10^{-20} \mathrm{~cm}^{2}$ \\
\hline 0.2 & 5.52 \\
0.137 & 6.21 \\
0.1 & 6.80 \\
0.05 & 7.87 \\
0.025 & 8.63 \\
EIA & 9.35 \\
B1B & 6.77 \\
\hline \hline
\end{tabular}

$0 \leqslant y \leqslant 11,-15 \leqslant z \leqslant 11$, which limits the application to the calculation of EC cross sections for $n<4$. However, these calculations illustrate the convergence of the GTDSE partial cross sections to the AOCC values for $n=2,3$; they are somewhat larger than the EIA results. For $n \geqslant 4$, the AOCC calculation probably overestimates the cross section as already explained for $E=100 \mathrm{keV} / \mathrm{u}$. As $n$ increases, the difficulty of augmenting the grid density precludes checking the convergence of the GTDSE calculation, but the good agreement among the GTDSE results for $n=5,6$ indicates a very small uncertainty of the grid calculations at high- $n$ levels. In fact, the G1 results are in agreement with the EIA values.

The convergence of the numerical calculation of the partial cross section $\sigma_{1}$ is particularly difficult to achieve because many grid points are required to accurately represent the wave function in a very small region close to the projectile nucleus. Nevertheless, it is possible to increase the grid density by keeping a modest box size for calculating this particular partial cross section. For this purpose we have carried out an exploratory calculation with the new grid $-5.5 \leqslant x \leqslant 5.5$, $0 \leqslant y \leqslant 5.5,-15 \leqslant z \leqslant 5.5$, and $\Delta_{q}=0.025 a_{0}$. The results are compared in Table $\mathrm{V}$ with the EIA value. A least-squares fitting of our cross sections to a polynomial of degree 2 of $\Delta_{q}^{-1}$ yields an extrapolated value of $9.3 \times 10^{-20} \mathrm{~cm}^{2}$ for $\Delta_{q}=0$, in good agreement with the EIA result, which is probably the most accurate value at this energy. As an additional check we have performed a first-order Coulomb-Born [46] (B1B in the notation of Ref. [46]) calculation of $\sigma_{1}$ using the grid representation of the initial and final wave functions. Although the accuracy of the B1B method is far from outstanding, the calculations performed with the low-density grids G1 and G2 have rapidly converged to the value shown in Table $\mathrm{V}$. This points to the kinetic energy operator, which is lacking in the $\mathrm{B} 1 \mathrm{~B}$ approximation, as responsible for the slow convergence of the complete GTDSE calculations.

\section{ELECTRON CAPTURE TOTAL CROSS SECTION}

The calculation of the EC total cross section in closecoupling and lattice methods is carried out by adding the partial cross sections for reactions (2). As explained in [19], the calculation of total cross sections requires the evaluation of the contribution of channels not included in the close-coupling basis set or which cannot be described by the limited extension of the box in lattice calculations. While this "topping-up" 


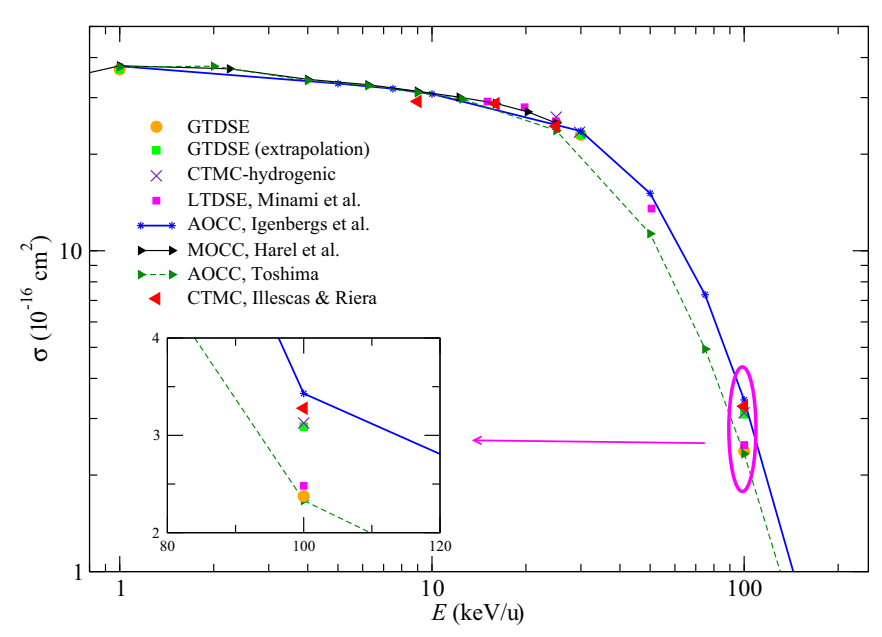

FIG. 5. Total cross section for electron capture in $\mathrm{Be}^{4+}+\mathrm{H}(1 s)$ collisions, as a function of the impact energy. (॰, $\square$ [orange dot, green square]), present GTDSE calculations; ( $\triangleleft)$, present CTMC-hydrogenic. Previous calculations: ( $\square$ [magenta square]), LTDSE [19], $(-*-)$, AOCC [20]; (- - ), AOCC [14]; (- -), MOCC [16].

effect is relatively unimportant at low energies, it strongly determines the accuracy of the calculated total cross sections at $E \approx 100 \mathrm{keV} / \mathrm{u}$. This point is illustrated in Fig. 5, where we compare some calculations of the EC total cross section. The two AOCC [14,20] disagree for energies higher than $25 \mathrm{keV} / \mathrm{u}$, as a consequence of the limited number of channels $(n \leqslant 5)$ included in the calculation of Toshima [14]. Although the calculation of Igenbergs et al. [20] includes up to $n=8$, the unphysical behavior of the partial cross sections in Fig. 3 casts some doubts on the accuracy of this calculation at $E \approx 100 \mathrm{keV} / \mathrm{u}$. Minami et al. [19] estimated the total cross section for $E \geqslant 50 \mathrm{keV} / \mathrm{u}$ by adding the partial cross sections from their lattice calculation for $n=1-5$ and the contributions from $n=6-10$ obtained using the $1 / n^{3}$ rule, which leads to a total cross section slightly higher than that of Toshima (see the inset of Fig. 5).

The EC total cross section is easily obtained in the CTMC calculations from the fraction of electron trajectories bound to the projectile at the end of the collision, and does not rely on the values of the partial cross sections, which are calculated afterwards using a partition of the phase space [47]. The total cross section from the GTDSE can be estimated as the sum of the partial cross sections for $n=1-8$ tabulated in Table IV, which lies between those of Toshima $\left(n_{\max }=5\right)$ and Minami et al. (extrapolated to $n_{\max }=10$ ). Inspection of Table IV and Fig. 3 shows that the GTDSE calculation can be easily extrapolated to the limit $n \rightarrow \infty$ using the CTMC-hydrogenic cross sections that follow the $n^{-3}$ rule; this extrapolation leads to a value of $3.11 \times 10^{-16} \mathrm{~cm}^{2}$ at $E=100 \mathrm{keV} / \mathrm{u}$, which is identical to the CTMC-hydrogenic cross section. The CTMC value contains two possible uncertainties. The first one is associated with the statistics employed. We have checked that the total cross section changes in about $10^{-18} \mathrm{~cm}^{2}$ when increasing the number of trajectories from $1 \times 10^{5}$ to $5 \times 10^{5}$. A more important uncertainty arises from the systematic error consequence of the overestimation of the transitions to low- $n$ levels. At $100 \mathrm{keV} / \mathrm{u}$, the overpopulation of the $n=2$ level can be estimated from Table IV and Fig. 3 in $1 \times 10^{-17} \mathrm{~cm}^{2}(\approx 3 \%$ of the total cross section). Therefore, the GTDSE calculation, with the CTMC "topping up" yields the most accurate total cross section value at $E=100 \mathrm{keV} / \mathrm{u}$ of $3.1 \times 10^{-16} \mathrm{~cm}^{2}$ with an uncertainty smaller than $10^{-17} \mathrm{~cm}^{2}$ by comparison with the CTMC cross section.

\section{SUMMARY}

In this work we have implemented a lattice method to solve numerically the time-dependent Schrödinger that arises in the eikonal treatment of ion-atom collisions and we have applied it to calculate $n$ partial and total electron capture cross sections for $\mathrm{Be}^{4+}+\mathrm{H}(1 s)$ collisions. The method can be applied in a wide energy range, although for energies below $1 \mathrm{keV} / \mathrm{u}$ trajectory effects start to be sizable, the semiclassical treatment is not appropriate, and the full quantum mechanical formalism must be applied. We have illustrated the workings of the method for four energies: $1,30,100$, and $500 \mathrm{keV} / \mathrm{u}$, where we have checked the convergence of the computational procedure, and we have compared the values of the $n$ partial cross sections with previous calculations.

A goal of the present calculation is to estimate the precision of the values of the cross sections, which are required in the applications. While it is difficult to quantify the uncertainties associated to the different calculation steps, the use of completely different computing schemes allows us to ensure that these uncertainties cannot be larger than the splitting between the computed cross sections. In this respect, we have been able to find agreement between two sets of values from different methodologies, and to give an estimate of their accuracy. For instance, at $E=1 \mathrm{keV} / \mathrm{u}$, the cross sections from AOCC and MOCC disagree by less than 5\%, and the differences with the GTDSE are on the order of $3 \%-6 \%$, which allows us to estimate the uncertainty of these data at about $5 \%$. At $E=30 \mathrm{keV} / \mathrm{u}$, the partial cross sections for $2 \leqslant n \leqslant 8$, calculated using AOCC and GTDSE, differ by less than $10 \%$. At $E=100 \mathrm{keV} / \mathrm{u}$, the GTDSE allows us to evaluate the accuracy of the CTMC results. As a conclusion, the CTMC-hydrogenic calculation provides accurate $n$ partial cross sections with the exception of the levels $n=1,2$. The cross sections for $n>2$ agree with the GTDSE ones with differences smaller than $15 \%$.

At $E=500 \mathrm{keV} / \mathrm{u}$, the convergence of the GTDSE calculation with the grid density is very slow, and the numerical calculation of the (very small) cross sections requires vast computational resources, in contrast to the requirements of other methods. Nevertheless, we have found good agreement for high- $n$ capture levels between GTDSE and EIA calculations, which are the most accurate values at this perturbative regime.

Finally, we have studied the convergence of the EC total cross section at $E=100 \mathrm{keV} / \mathrm{u}$, where important discrepancies remain. We find that the partial cross sections from the GTDSE method can be extrapolated using the CTMChydrogenic results for high $n$ to obtain a value of the total cross section whose uncertainty is smaller than $10^{-17} \mathrm{~cm}^{2}$. In conclusion, with the available computational resources, the numerical solution of the time-dependent Schrödinger equation is a valuable tool to calculate electron capture cross 
sections in ion-atom collisions at intermediate energies, where these data are required for applications in fusion plasmas.

\section{ACKNOWLEDGMENTS}

This work has been partially supported by the Ministerio de Economía y Competitividad (Spain) (Projects
No. ENE2011-28200 and No. ENE2014-52432-R). Calculations have been performed at the Centro de Computación Científica (UAM, Madrid) and at the Barcelona Supercomputing Center (BSC) with financial support from the Red Española de Supercomputación (RES; Grants No. FI-2012-2-0016, No. FI-2013-1-0020, and No. FI2013-2-0006). We thank Bernard Pons for helpful discussions.
[1] R. Pitts, S. Carpentier, F. Escourbiac, T. Hirai, V. Komarov, A. Kukushkin, S. Lisgo, A. Loarte, M. Merola, R. Mitteau, A. Raffray, M. Shimada, and P. Stangeby, J. Nucl. Mater. 415, S957 (2011).

[2] J. Paméla, G. Matthews, V. Philipps, and R. Kamendje, J. Nucl. Mater. 363, 1 (2007).

[3] R. Neu, G. Arnoux, M. Beurskens, V. Bobkov, S. Brezinsek, J. Bucalossi, G. Calabro, C. Challis, J. W. Coenen, E. de la Luna, P. C. de Vries, R. Dux, L. Frassinetti, C. Giroud, M. Groth, J. Hobirk, E. Joffrin, P. Lang, M. Lehnen, E. Lerche, T. Loarer, P. Lomas, G. Maddison, C. Maggi, G. Matthews, S. Marsen, M.-L. Mayoral, A. Meigs, P. Mertens, I. Nunes, V. Philipps, T. Pütterich, F. Rimini, M. Sertoli, B. Sieglin, A. C. C. Sips, D. van Eester, G. van Rooij, and J.-E. Contributors, Phys. Plasmas 20, 056111 (2013).

[4] R. C. Isler, Plasma Phys. Controlled Fusion 36, 171 (1994).

[5] B. Schunke, D. Bora, R. Hemsworth, and A. Tanga, AIP Conf. Proc. 1097, 480 (2009).

[6] W. Biel, T. Baross, P. Bourauel, D. Dunai, M. Durkut, G. Erdei, N. Hawkes, M. Hellermann, A. Hogenbirk, R. Jaspers, G. Kiss, F. Klinkhamer, J. Koning, V. Kotov, Y. Krasikov, A. Krimmer, O. Lischtschenko, A. Litnovsky, O. Marchuk, O. Neubauer, G. Offermanns, A. Panin, K. Patel, G. Pokol, M. Schrader, B. Snijders, V. Szabo, N. van der Valk, R. Voinchet, J. Wolters, and S. Zoletnik, Fusion Eng. Des. 86, 548 (2011).

[7] H. J. Ludde and R. M. Dreizler, J. Phys. B 15, 2713 (1982).

[8] T. O. Yamaguchi and Y. Itikawa, J. Phys. Soc. Jpn. 51, 2982 (1982).

[9] L. Errea, L. Méndez, and A. Riera, Phys. Lett. A 92, 231 (1982).

[10] W. Fritsch and C. D. Lin, Phys. Rev. A 29, 3039 (1984).

[11] H. Suzuki, N. Toshima, T. Watanabe, and H. Ryufuku, Phys. Rev. A 29, 529 (1984).

[12] D. Belkić, S. Saini, and H. S. Taylor, Phys. Rev. A 36, 1601 (1987).

[13] D. Belkić, R. Gayet, and A. Salin, At. Data Nucl. Data Tables 51, 59 (1992).

[14] N. Toshima, Phys. Rev. A 50, 3940 (1994).

[15] L. F. Errea, C. Harel, H. Jouin, L. Méndez, B. Pons, and A. Riera, J. Phys. B 31, 3527 (1998).

[16] C. Harel, H. Jouin, and B. Pons, At. Data. Nucl. Data Tables 68, 279 (1998).

[17] C. Illescas and A. Riera, Phys. Rev. A 60, 4546 (1999).

[18] A. T. Le, M. Hesse, T. G. Lee, and C. D. Lin, J. Phys. B 36, 3281 (2003).

[19] T. Minami, M. S. Pindzola, T.-G. Lee, and D. R. Schultz, J. Phys. B 39, 2877 (2006).

[20] K. Igenbergs, J. Schweinzer, and F. Aumayr, J. Phys. B 42, 235206 (2009).

[21] I. Y. Tolstikhina, M. S. Litsarev, D. Kato, M.-Y. Song, J.-S. Yoon, and V. P. Shevelko, J. Phys. B 47, 035206 (2014).
[22] H. K. Chung and B. J. Braams, J. Phys.: Conf. Ser. 635, 052020 (2015).

[23] H.-K. Chung, B. J. Braams, K. Bartschat, A. G. Császár, G. W. F. Drake, T. Kirchner, V. Kokoouline, and J. Tennyson, J. Phys. D: Appl. Phys. 49, 363002 (2016).

[24] The Editors, Phys. Rev. A 83, 040001 (2011).

[25] K. Bartschat and O. Zatsarinny, Phys. Scr. 90, 054006 (2015)

[26] J. Suarez, S. Farantos, S. Stamatiadis, and L. Lathouwers, Comput. Phys. Commun. 180, 2025 (2009).

[27] L. Errea, C. Illescas, L. Méndez, I. Rabadán, and J. Suárez, Chem. Phys. 462, 17 (2015).

[28] R. Abrines and I. C. Percival, Proc. Phys. Soc. 88, 861 (1966).

[29] D. J. W. Hardie and R. E. Olson, J. Phys. B 16, 1983 (1983).

[30] J. S. Cohen, J. Phys. B 18, 1759 (1985).

[31] M. J. Raković, D. R. Schultz, P. C. Stancil, and R. K. Janev, J. Phys. A: Math. Gen. 34, 4753 (2001).

[32] N. D. Cariatore, S. Otranto, and R. E. Olson, Phys. Rev. A 91, 042709 (2015).

[33] A. Jorge, L. F. Errea, C. Illescas, and L. Méndez, Phys. Rev. A 93, 066701 (2016).

[34] N. D. Cariatore, S. Otranto, and R. E. Olson, Phys. Rev. A 93, 066702 (2016).

[35] R. Guantes and S. C. Farantos, J. Chem. Phys. 111, 10827 (1999).

[36] C. Leforestier, R. H. Bisseling, C. Cerjan, M. D. Feit, R. Friesner, A. Guldberg, A. Hammerich, G. Jolicard, W. Karrlein, H.-D. Meyer, N. Lipkin, O. Roncero, and R. Kosloff, J. Comput. Phys. 94, 59 (1991).

[37] D. Dundas, J. F. McCann, J. S. Parker, and K. T. Taylor, J. Phys. B 33, 3261 (2000).

[38] R. Kosloff and D. Kosloff, J. Comput. Phys. 63, 363 (1986).

[39] U. D. Giovannini, A. H. Larsen, and A. Rubio, Eur. Phys. J. B 88, 56 (2015).

[40] C. Harel, H. Jouin, B. Pons, L. F. Errea, L. Méndez, and A. Riera, Phys. Rev. A 55, 287 (1997).

[41] K. Igenbergs, Calculation of cross sections relevant for diagnostics of hot fusion plasmas, Ph.D. thesis, Technischen Universität Wien, 2011.

[42] L. F. Errea, C. Illescas, L. Méndez, B. Pons, A. Riera, and J. Suárez, Phys. Rev. A 70, 052713 (2004).

[43] A. Jorge, L. Errea, C. Illescas, and L. Méndez, Eur. Phys. J. D 68, 227 (2014).

[44] M. S. Gravielle and J. E. Miraglia, Phys. Rev. A 51, 2131 (1995).

[45] A. Jorge, C. Illescas, J. E. Miraglia, and M. S. Gravielle, J. Phys. B 48, 235201 (2015).

[46] D. P. Dewangan and J. Eichler, J. Phys. B: At. Mol. Phys. 19, 2939 (1986).

[47] R. L. Becker and A. D. MacKellar, J. Phys. B 17, 3923 (1984). 